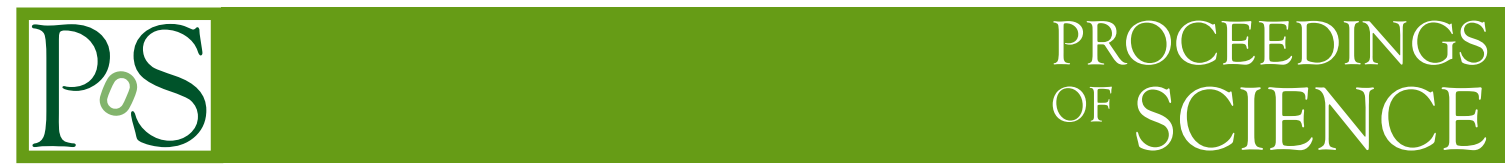

\title{
Hadron Physics Experimental Overview
}

\section{Beijiang Liu*}

Institute of High Energy Physics, Chinese Academy of Sciences

E-mail: liubj@ihep.ac.cn

The sets of data presently available from high energy frontier and high intensity frontier offer great opportunities to test both perturbative and non-perturbative aspects of Quantum Chromodynamics. A selection of hadron physics results are reviewed.

38th International Conference on High Energy Physics 3-10 August 2016

Chicago, USA

${ }^{*}$ Speaker. 


\section{Introduction}

Quantum Chromodynamics (QCD) is the underlying fundamental theory for strong interaction, which is unique and remains challenging in the standard model. In the high energy regime, the asymptotic freedom of the partons constituting hadrons, allows to treat the interaction with perturbative models. In the low energy regime, the non-Abelian character of QCD requires a nonperturbative approach which must rely either on Lattice QCD or on QCD-inspired models.

Heavy-flavour production in high energy hadronic collisions, which provides an important testing ground for both perturbative and non-perturbative aspects of QCD calculations. The heavyquark mass acts as a long distance cut-off so that the hard-scattering process can be calculated with perturbative QCD (pQCD) down to low transverse momenta. The heavy-quark pair forms a quarkonium bound state, on the other hand, is non-perturbative. Furthermore, the quality of effective models describing strong interaction are crucial to predict and model the backgrounds for precise measurements and discoveries. Recent results from Large Hadron Collider (LHC) experiments are discussed in Section 2.

Hadron spectroscopy is a unique way to access long-distance regime of QCD which remains the least understood aspect of the theory. QCD-motivated models for hadrons predict "Exotic Hadrons" that have structures that are more complex than the quark-antiquark mesons and threequark baryons of the original quark model, such as glueballs, hybridsand multi-quarkstates. Experimental search of these predictions and subsequent investigation of their properties would provide validation of and valuable input to the quantitative understanding of QCD. New progress and discoveries are discussed in Section 3.

\section{Heavy-flavour and quarkonium production}

The theoretical description of the heavy quark production mechanisms and their agreement with the experimental data has seen a significant improvement in the last years. Both in the charm and in the beauty sectors, the recent measurements at $\sqrt{s}=13 \mathrm{TeV}$ performed by the LHC experiments are found in reasonable agreement with predictions [1].

For charmonium production, the theoretical expectations were found in good agreement with the experimental data of the ATLAS, CMS, and LHCb collaborations [2, 3, 4]. The latest results of LHC data extended the $\Upsilon$ production cross-section measurement at the increased energy of $\sqrt{s}=13$ $\mathrm{TeV}$. Each result at a different energy scale and precision can provide useful constraint on the process models. The latest measurement from CMS shows that the production cross-section scales linearly with the energy (Figure 1), as naively expected. The same behavior was also observed, for example, for the production of $c \bar{c}$ or b-hadrons [3]. Precise comparison with the expectations awaits for the update of the theoretical models [5]. The recent study of $b \bar{b}$ production from the study of b-hadron semileptonic decays at $13 \mathrm{TeV}$ shows some tension with theoretical predictions, especially at low $\eta[6]$, which call for further investigation.

Differential cross sections are presented for the prompt and non-prompt production of the hidden-charm states $X(3872)$ and $\psi(2 S)$ measured at $\sqrt{s}=8 \mathrm{TeV}$ by the ATLAS experiment [7]. The prompt $X(3872)$ cross-section measurement shows good agreement with the CMS result. Good agreement is found with theoretical predictions within the model based on NLO NRQCD, 

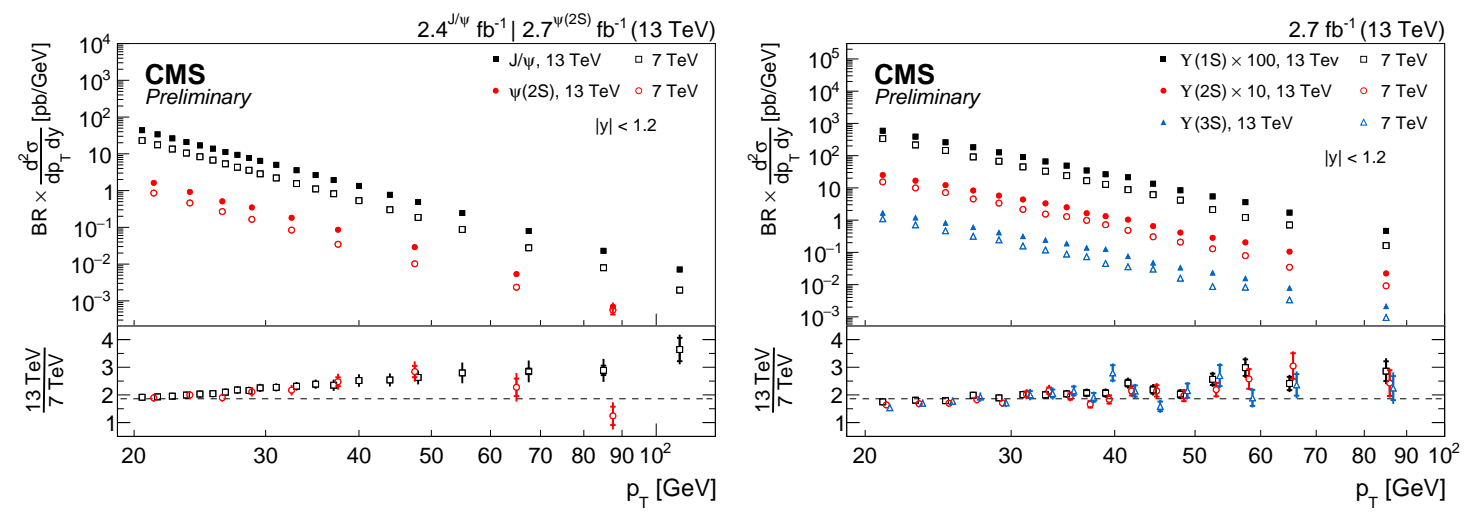

Figure 1: Measurement of the $J / \psi$ and $\psi(2 S)$ production cross-sections (a) and $\Upsilon$ production cross-sections (b) in pp collisions at $\sqrt{s}=13 \mathrm{TeV}$ performed by the CMS Collaborations.

which considers $X(3872)$ to be a mixture of $\chi_{c 1}(2 P)$ and a $D^{0} \bar{D}^{* 0}$ molecular state, with the production being dominated by the $\chi_{c 1}(2 P)$ component and the normalisation fixed through the fit to CMS data. The non-prompt production of $\psi(2 S)$ is described by the FONLL predictions within the uncertainties (Figure 2 ). But the same predictions, recalculated for $X(3872)$ using the branching fraction extracted from the Tevatron data, overestimate the non-prompt production of $X(3872)$, especially at large transverse momenta (Figure 2 ). Assuming independent single effective lifetimes for non-prompt $X(3872)$ and $\psi(2 S)$ production gives $R_{B}=\frac{\mathscr{B}(B \rightarrow X(3872)+\text { any }) \mathscr{B}\left(X(3872) \rightarrow J / \psi \pi^{+} \pi^{-}\right)}{\mathscr{B}(B \rightarrow \psi(2 S)+\text { any }) \mathscr{B}\left(\psi(2 S) \rightarrow J / \psi \pi^{+} \pi^{-}\right)}=$ (3.95 \pm 0.32 (stat) \pm 0.08 (sys) $) \times 10^{-2}$, while separating short- and long-lived contributions, assuming that the short-lived component is due to $B_{c}$ decays, gives $R_{B}=(3.57 \pm 0.33$ (stat) \pm 0.11 (sys) $) \times$ $10^{-2}$, with the fraction of non-prompt $X(3872)$ produced via $B_{c}$ decays for $p_{\mathrm{T}}(X(3872))>10 \mathrm{GeV}$ being $(25 \pm 13$ (stat) \pm 2 (sys) \pm 5 (spin) $) \%$.

\section{Hadron spectroscopy}

Although years of continuous experimental efforts have been made to search for QCD exotic hadrons beyond quark model, no compelling evidence has been unambiguously established yet. However, strong evidence for mesons that do not fit into the simple $q \bar{q}$ scheme of the original quark model has been steadily accumulating during the past decade. Most recently, a comprehensive review covers in great detail both experimental and theoretical aspects of the heavy-quark QCD exotica [8].

\subsection{The $P_{c}$ Pentaquark Candidates}

LHCb performed a full amplitude analysis of the process $\Lambda_{b} \rightarrow K(p J / \psi)$, which included all known $\Lambda$ states decaying to $K p$ [9]. Two additional amplitudes in the $p J / \psi$ system were needed to describe the data, both found with more than $9 \sigma$ significance (Fig. 3). The lighter one, the $P_{c}(4380)$, was wide, with a width around $200 \mathrm{MeV}$; the heavier one, the $P_{c}(4450)$, was narrow, with a width around $40 \mathrm{MeV}$. The favored $J^{P}$ of the $P_{c}(4380)$ and $P_{c}(4450)$ were found to be $\frac{3}{2}^{-}$and $\frac{5}{2}^{+}$, respectively, although the combinations $\left(\frac{3}{2}^{+}, \frac{5}{2}^{-}\right)$and $\left(\frac{5}{2}^{+}, \frac{3}{2}^{-}\right)$could not be ruled out. The phase motion indicated by the Argand diagram for the narrower $P_{c}(4450)$ was found to be consistent with 

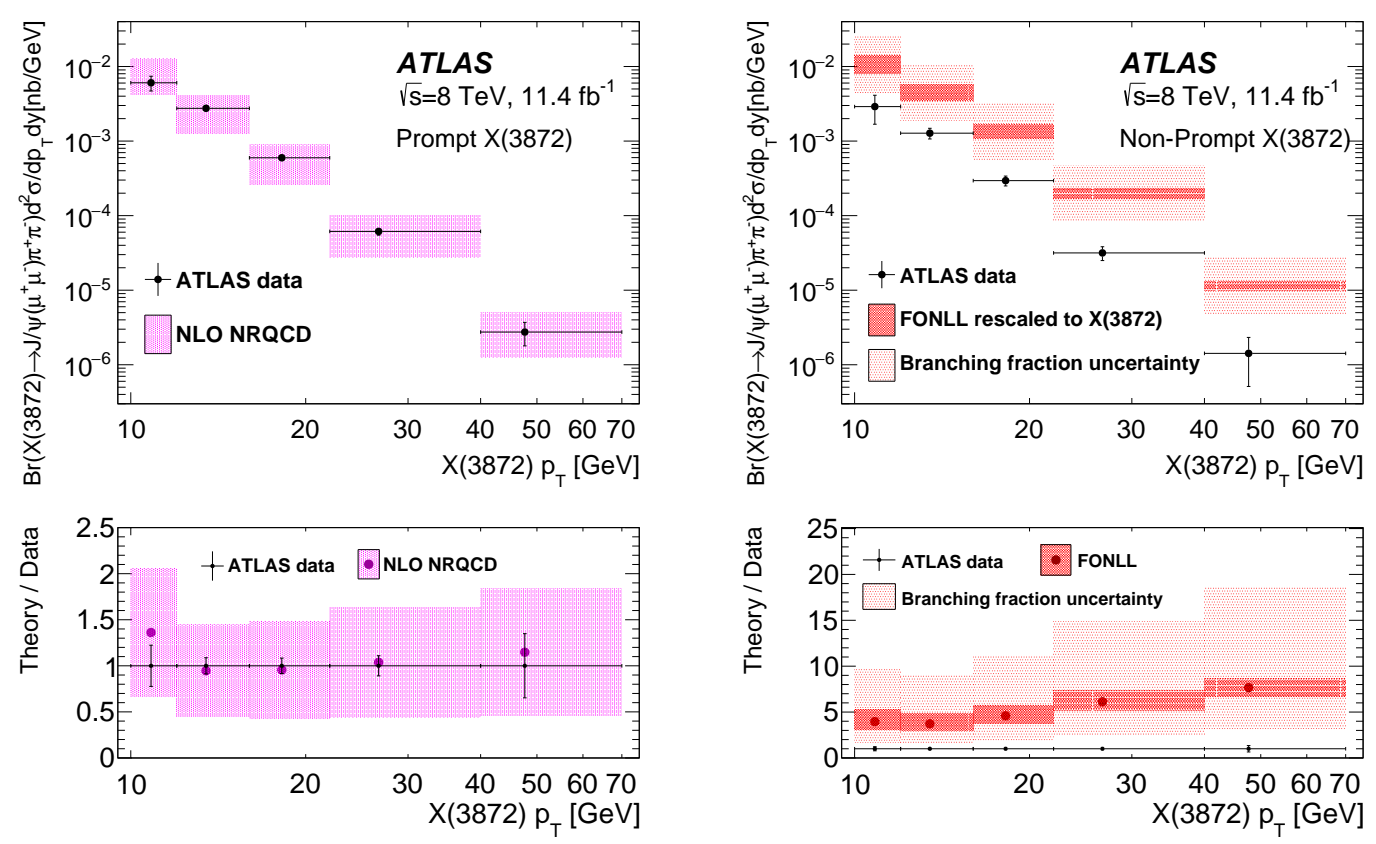

Figure 2: Measured cross section times branching fractions as a function of $p_{T}$ for (a) prompt $X(3872)$ compared to NLO NRQCD predictions with the $X(3872)$ modeled as a mixture of $\chi_{c 1}(2 P)$ and a $D^{0} \bar{D}^{* 0}$ molecular state, and (b) non-prompt $X(3872)$ compared to the FONLL model prediction.

a resonance; the Argand diagram for the wider $P_{c}(4380)$ was more uncertain and depends more upon the details of the $p K$ amplitudes, which are not precisely known. LHCb also performed an analysis of the Cabibbo-suppressed decay $\Lambda_{b} \rightarrow \pi(p J / \psi)$ was performed [10]. A significantly better description of the data is achieved when, in addition to the previously observed nucleon excitations $N \rightarrow p \pi^{-}$, either the $P_{c}(4380)^{+}$and $P_{c}(4450)^{+} \rightarrow J / \psi p$ states, previously observed in $\Lambda_{b} \rightarrow J / \psi p K^{-}$decays, or the $Z_{c}(4200)^{-} \rightarrow J / \psi \pi^{-}$state, previously reported in $B^{0} \rightarrow J / \psi K^{+} \pi^{-}$ decays, or all three, are included in the amplitude models. The data support a model containing all three exotic states, with a significance of more than three standard deviations. Within uncertainties, the data are consistent with the $P_{c}(4380)^{+}$and $P_{c}(4450)^{+}$production rates expected from their previous observation taking account of Cabibbo suppression.

\section{2 $B \rightarrow K \phi J / \psi$ and the $Y(4140)$ and More}

The $Y(4140)$ was first reported in 2009 by the CDF Collaboration in the process $B \rightarrow K Y$ with $Y \rightarrow \phi J / \psi$ [11]. A series of positive [12,13] and negative [14, 15] searches using the same process followed, making the status of the $Y$ (4140) uncertain. In addition to the $Y(4140)$, the CDF and CMS Collaborations found evidence for a higher-mass structure, the $Y(4274)$ [13, 16], whose status was also uncertain.

Recently, a higher-statistics analysis from the LHCb experiment was performed [17, 18]. The existence of the $Y(4140)$ and the $Y(4274)$ is confirmed with significances of $8.4 \sigma$ and $6.0 \sigma$, respectively, although the width of $Y(4140)$ is substantially larger. Further more, the $X(4500)$ and 

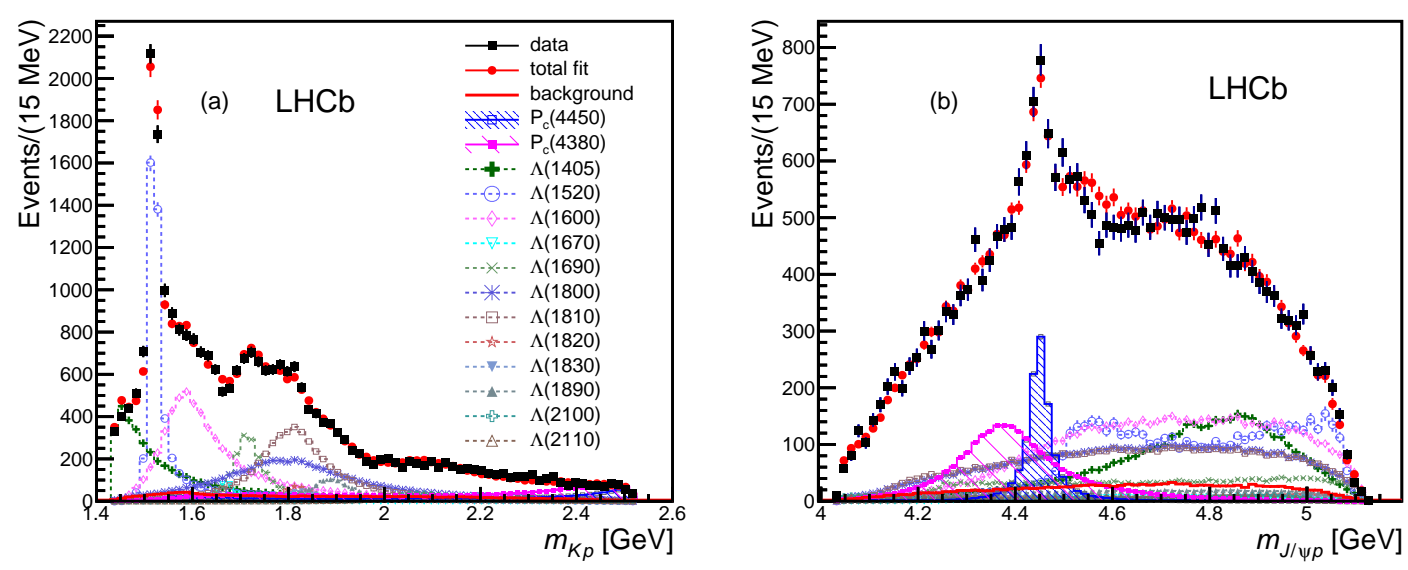

Figure 3: Observation of the $P_{c}(4380)$ and $P_{c}(4450)$ by LHCb in $\Lambda_{b} \rightarrow K(p J / \psi)$.

$X(4700)$, with significances greater than $5 \sigma$ (Fig. 4) are reported. Using a full six-dimensional amplitude analysis, including $K^{*}$ resonances in the $K \phi$ system and descriptions of all decay angular distributions, the $J^{P C}$ of the $Y(4140)$ and the $Y(4274)$ were both determined to be $1^{++}$. The $J^{P C}$ values of the higher-mass $X(4500)$ and $X(4700)$ were both found to be $0^{++}$.

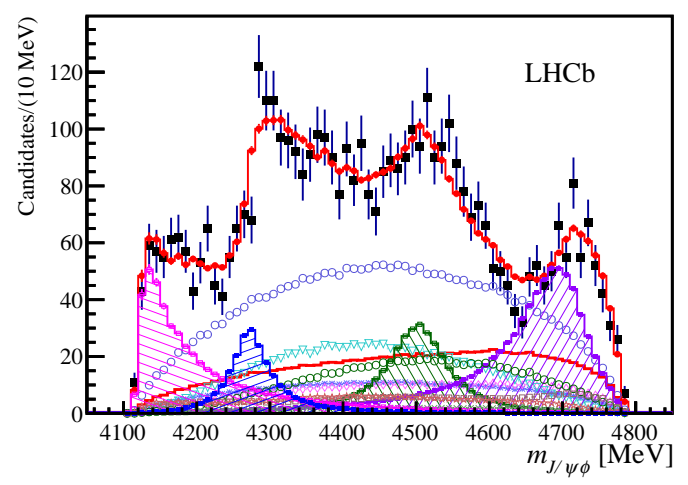

Figure 4: Observation of the $Y(4140), Y(4274), X(4500)$, and $X(4700)$ by LHCb in $B \rightarrow K(\phi J / \psi)$.

\subsection{Charmoniumlike mesons in $e^{+} e^{-}$annihilation}

The charmonium spectrum below the open charm threshold are in good agreement with the prediction of charm and anti-charm potential model. However, above the open charm threshold, the $e^{+} e^{-}$cross sections show many puzzling features. The BESIII measurement of the $e^{+} e^{-} \rightarrow$ $\pi^{+} \pi^{-} J / \psi$ cross section [19] is shown in Fig. 5(a). The peak that was formerly known as the $Y(4260)$ can be better described with two peaks, a narrow peak around $4.23 \mathrm{GeV}$ and a much wider peak at higher mass. Similarly, in $e^{+} e^{-} \rightarrow \pi^{+} \pi^{-} h_{c}(1 P)$, the data is also clearly inconsistent with a $Y(4260)$; there is some evidence for a narrow peak around $4.23 \mathrm{GeV}$ and a much wider peak at higher mass (Fig. 5(b)) [20]. The $\omega \chi_{c 0}$ cross section also shows evidence for peaking at a mass lower than that of the $Y(4260)$, a feature that has been named the $Y(4230)$ [21]. Other cross sections, such as $\eta J / \psi$ [22], $\omega \chi_{c 1,2}$ [23], have also proved to be remarkably complex. 

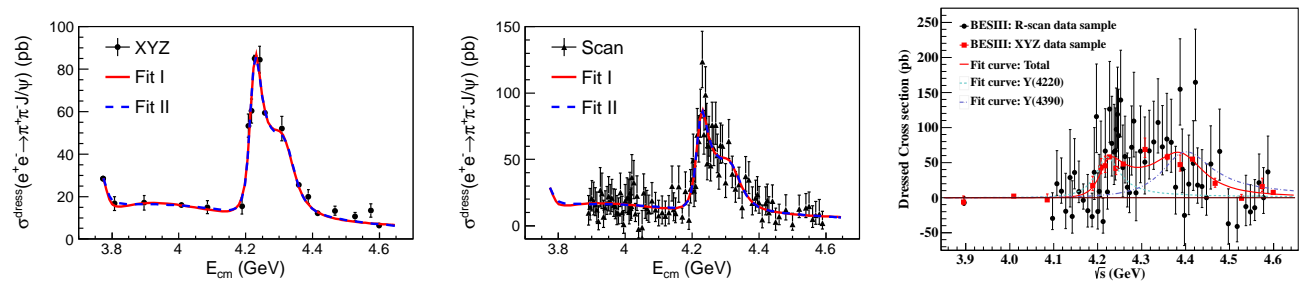

Figure 5: Measurements of $e^{+} e^{-} \rightarrow \pi^{+} \pi^{-} J / \psi$ (a) and $e^{+} e^{-} \rightarrow \pi^{+} \pi^{-} h_{c}$ (b) cross sections at BESIII.

In the study of $e^{+} e^{-} \rightarrow J / \psi \pi^{+} \pi^{-}$, a distinct charged structure, named the $Z_{c}(3900)^{ \pm}$, was observed in the $J / \psi \pi^{ \pm}$spectrum by BESIII [24] and Belle [25] in 2013. Its existence was confirmed shortly thereafter with CLEO-c data [26]. Shortly after the discovery of the $Z_{c}(3900)$, the BESIII experiment did observe a narrow peak (with a width of roughly $8 \mathrm{MeV}), Z_{c}(4020)$, in the $\pi^{ \pm} h_{c}(1 P)$ mass spectrum near the $D^{*} \bar{D}^{*}$ threshold [27]. However, no evidence for the $Z_{c}(3900) \rightarrow \pi^{ \pm} h_{c}(1 P)$ nor $Z_{c}(4020) \rightarrow \pi^{ \pm} J / \psi$ could be found.

The BESIII experiment also studied the $Z_{c}(3900)$ and $Z_{c}(4020)$ in open-charm decays. The $Z_{c}(3900)$ was found to decay to $D \bar{D}^{*}$ in the process $e^{+} e^{-} \rightarrow D \bar{D}^{*} \pi[28,29]$. The $Z_{c}(4020)$ was found in the process $e^{+} e^{-} \rightarrow D^{*} \bar{D}^{*} \pi$ decaying to $D^{*} \bar{D}^{*}$ [30]. Similar to bottomonium, the decays of the $Z_{c}(3900)$ and $Z_{c}(4020)$ to open charm are roughly an order of magnitude larger than their decays to closed charm. It is noticed that the $Z_{c}(3900)$ is lighter and narrower in its opencharm decay, while the $Z_{c}(4020)$ is heavier and wider in its open-charm decay. Neutral partners to the $Z_{c}(3900)$ and $Z_{c}(4020)$ were subsequently discovered in the neutral versions of all four reactions listed above, thus complementing the isospin-triplet representation of isospin one, $I=1$, resonances. The $Z_{c}(3900)$ was found to decay to $\pi^{0} J / \psi[26,31]$ and $\left(D \bar{D}^{*}\right)^{0}$ [32]; the $Z_{c}(4020)$ was found to decay to $\pi^{0} h_{c}(1 P)$ [33] and $\left(D^{*} \bar{D}^{*}\right)^{0}$ [34]. It is interesting to note that neither the $Z_{c}(3900)$ nor the $Z_{c}(4020)$ has been seen in $B$ decays.
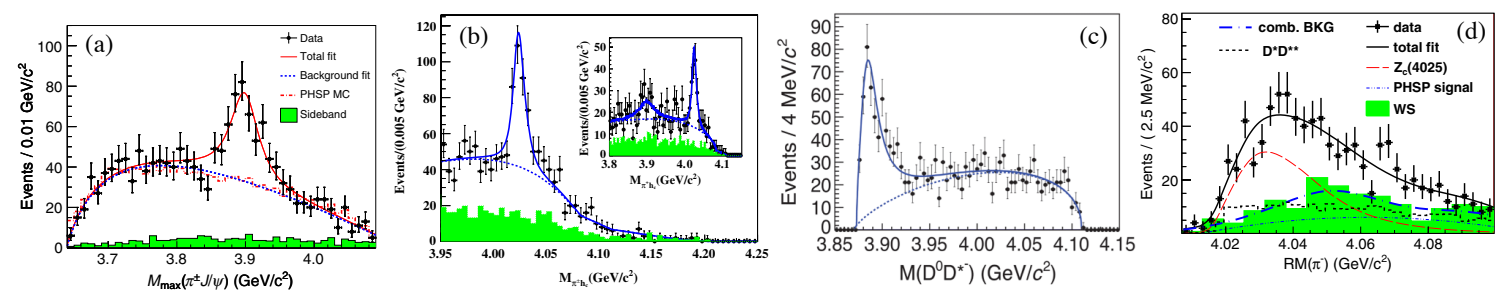

Figure 6: The $Z_{c}$ states observed in $e^{+} e^{-}$annihilation in the charmonium region. (a) Observation of the $Z_{c}$ (3900) in $e^{+} e^{-} \rightarrow \pi^{\mp} Z_{c}$ with the $Z_{c}$ decaying to $\pi^{ \pm} J / \psi$ [24]. (b) Observation of the $Z_{c}(4020)$ in $e^{+} e^{-} \rightarrow \pi^{\mp} Z_{c}$ with the $Z_{c}$ decaying to $\pi^{ \pm} h_{c}(1 P)$ [27]. (c) Observation of the $Z_{c}(3900)$ decaying to $\left(D \bar{D}^{*}\right)^{ \pm}$[28]. (d) Observation of the $Z_{c}(4020)$ decaying to $\left(D^{*} \bar{D}^{*}\right)^{ \pm}$[30]. All figures are from the BESIII experiment.

\subsection{Bottomoniumlike mesons in $e^{+} e^{-}$annihilation}

Recently, Belle used a increased number of center-of-mass energy points to map out the region of the $\Upsilon(5 S)$ and $\Upsilon(6 S)$ [35]. Two peaks could be seen clearly in the exclusive $e^{+} e^{-} \rightarrow$ 
$\pi^{+} \pi^{-} \Upsilon(n S)$ cross sections, and with negligible backgrounds. The fit to the $R_{b}$ spectrum yielded consistent parameters, but the interference with the non-resonant $b \bar{b}$ continuum makes the fits to the $R_{b}$ spectrum unreliable. The same two peaks are also apparent in the exclusive cross sections for $e^{+} e^{-} \rightarrow \pi^{+} \pi^{-} h_{b}(n P) n=1,2$, with little nonresonant background (Fig. 7c) [36]. The sizes of the cross sections are similar to those for $e^{+} e^{-} \rightarrow \pi^{+} \pi^{-} \Upsilon(n S)$. Recently, the Belle experiment reported a clear $e^{+} e^{-} \rightarrow \Upsilon(5 S) \rightarrow B_{s}^{(*)} \bar{B}_{s}^{(*)}$ signal, with no statistically significant signal of $e^{+} e^{-} \rightarrow \Upsilon(11020) \rightarrow B_{s}^{(*)} \bar{B}_{s}^{(*)}[37]$.
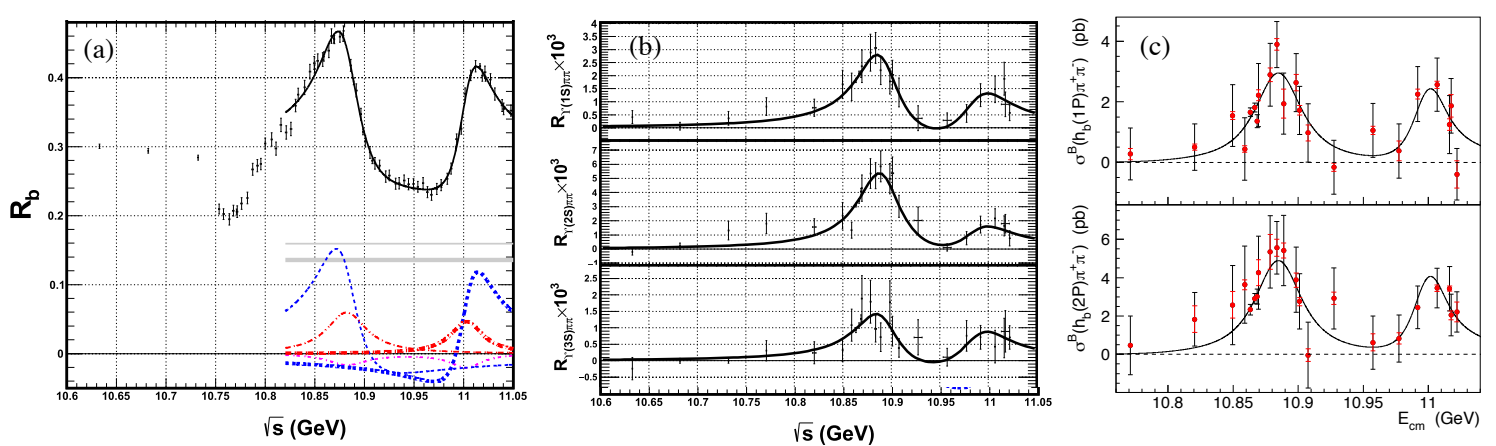

Figure 7: The $\Upsilon(5 S)$ and $\Upsilon(6 S)$ observed at Belle. (a) The inclusive $e^{+} e^{-}$cross section (shown as $R_{b} \equiv$ $\left.\sigma(b \bar{b}) / \sigma_{\mu \mu}^{0}\right)$. The solid lines are for a fit that includes interfering $\Upsilon(5 S)$ and $\Upsilon(6 S)$ states as well as coherent and incoherent backgrounds [35]. (b) The exclusive $e^{+} e^{-} \rightarrow \pi^{+} \pi^{-} \Upsilon(1 S, 2 S, 3 S)$ cross sections [35]. (c) The exclusive $e^{+} e^{-} \rightarrow \pi^{+} \pi^{-} h_{b}(1 P, 2 P)$ cross sections [36].

It is very interesting to notice that $e^{+} e^{-} \rightarrow \pi^{+} \pi^{-} \Upsilon(1 S, 2 S, 3 S)$ and $e^{+} e^{-} \rightarrow \pi^{+} \pi^{-} h_{b}(1 P, 2 P)$ at center-of-mass energies near the $\Upsilon(5 S)$ mass proceed, either entirely or partially, through the intermediate processes $e^{+} e^{-} \rightarrow \pi^{ \pm} Z_{b}(10610)$ and $e^{+} e^{-} \rightarrow \pi^{ \pm} Z_{b}(10650)$, where the $Z_{b}(10610)$ and $Z_{b}(10650)$ are electrically charged, have widths on the order of $20 \mathrm{MeV}$, and decay to $\pi^{\mp} \Upsilon(1 S, 2 S, 3 S)$ and $\pi^{\mp} h_{b}(1 P, 2 P)$ [38]. A six-dimensional amplitude analysis of the $e^{+} e^{-} \rightarrow \pi^{+} \pi^{-} \Upsilon(1 S, 2 S, 3 S)$ processes was performed [39]. The $J^{P}=1^{+}$hypothesis was favored for both the $Z_{b}(10610)$ and the $Z_{b}(10650) . Z_{b}(10610)$ and $Z_{b}(10650)$ are just above the thresholds of $B \bar{B}^{*}$ and $B^{*} \bar{B}^{*}$, respectively. The Belle experiment observed the decays $Z_{b}(10610) \rightarrow B \bar{B}^{*}$ and $Z_{b}(10650) \rightarrow B^{*} \bar{B}^{*}$ [40], shown in Fig. 8d. No evidence was found for $Z_{b}(10650) \rightarrow B \bar{B}^{*}$ decay, and no evidence was found for the process $e^{+} e^{-} \rightarrow B \bar{B} \pi$. Assuming the charged $Z_{b}(10610)$ and $Z_{b}(10650)$ decay only to $\pi^{ \pm} \Upsilon(1 S, 2 S, 3 S), \pi^{ \pm} h_{b}(1 P, 2 P)$, and $B \bar{B}^{(*)}$ (which is supported by the study of the inclusive $\Upsilon(5 S)$ cross section [35]), branching fractions could be calculated. It was found that the open-bottom decays are roughly an order of magnitude larger than the closed-bottom decays.

\subsection{The issue of the $X(5568)$}

The issue of the $X(5568)$, recently reported by the $\mathrm{D} 0$ experiment in inclusive $p \bar{p}$ production at a center-of-mass energy of $1.96 \mathrm{TeV}$ [41], also remains unsettled. Because it decays to $B_{s} \pi^{ \pm}$, it could be a tetraquark state of $b, s, u$, and $d$. It could be related to the charged $Z_{c}$ or the $Z_{b}$, but it differs in the fact that its mass is significantly below the threshold of $B$ and a $K$, while the $Z_{c}$ and $Z_{b}$ states have masses above the open-charm and open-bottom thresholds, respectively. The D0 experiment reported that a significant fraction (around 10\%) of the $B_{s}$ produced in the transverse 

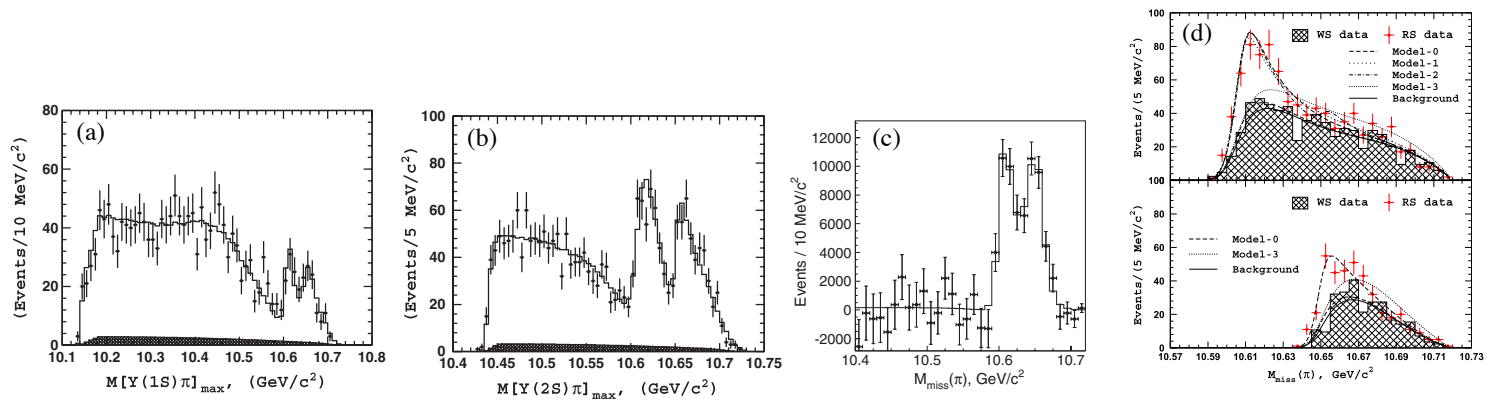

Figure 8: The $Z_{b}$ states observed in $e^{+} e^{-}$annihilation in the bottomonium region. (a,b,c) Observation of the $Z_{b}(10610)$ and $Z_{b}(10650)$ in $e^{+} e^{-} \rightarrow \pi^{\mp} Z_{b}$ with the $Z_{b}$ decaying to $\pi^{ \pm} \Upsilon(1 S)$ (a), $\pi^{ \pm} \Upsilon(2 S)$ (b), and $\pi^{ \pm} h_{b}(1 P)$ (c) [38]. (d) Observation of the $Z_{b}(10610)$ decaying to $\left(B \bar{B}^{*}\right)^{ \pm}$(top) and the $Z_{b}(10650)$ decaying to $\left(B^{*} \bar{B}^{*}\right)^{ \pm}$(bottom) [40].

momentum region between 10 and $30 \mathrm{GeV}$ originated from $X(5568)$ decays. The D0 experiment recently found an enhancement in $M\left(B_{s}^{0} \pi^{ \pm}\right)$with $B_{s}^{0} \rightarrow D_{s} \mu v$ at the same mass and at the expected width and rate [42]. The LHCb experiment and CMS experiment searched for the same state, but with $p p$ collisions and with center-of-mass energies at 7 and $8 \mathrm{TeV}$, but found no evidence for it $[43,44]$. The $X(5568)$ certainly deserves further study.

\subsection{A candidate of $p \bar{p})$ bound state in $J / \psi$ radiative decays}

The state $X(1835)$ was first observed by the BESII experiment as a peak in $J / \psi \rightarrow \gamma \eta^{\prime} \pi^{+} \pi^{-}$ decays [45]. This observation was later confirmed by BESIII [46] and was also observed in the $\eta K_{S}^{0} K_{S}^{0}$ channel, where its spin-parity was determined to be $J^{P}=0^{-}$by a partial wave analysis (PWA) [47]. An anomalously strong enhancement at the proton-antiproton $(p \bar{p})$ mass threshold, dubbed $X(p \bar{p})$, was first observed by BESII in $J / \psi \rightarrow \gamma p \bar{p}$ decays [48]; this observation was confirmed by BESIII [49] and CLEO [50]. This enhancement structure was subsequently determined to have spin-parity $J^{P}=0^{-}$by BESIII [51]. Using high statistics $J / \psi$ events, BESIII studies the $J / \psi \rightarrow \gamma \eta^{\prime} \pi^{+} \pi^{-}$process and observe a significant abrupt change in the slope of the $\eta^{\prime} \pi^{+} \pi^{-}$invariant mass distribution at the proton-antiproton $(p \bar{p})$ mass threshold [52]. Two models are used to characterize the $\eta^{\prime} \pi^{+} \pi^{-}$line shape around $1.85 \mathrm{GeV} / c^{2}$ : one which explicitly incorporates the opening of a decay threshold in the mass spectrum (Flatté formula) (Figure 9(a)), and another which is the coherent sum of two resonant amplitudes(Figure 9 (b)). Both fits show almost equally good agreement with data, and suggest the existence of either a broad state with strong couplings to $p \bar{p}$ final states or a narrow state just below the $p \bar{p}$ mass threshold. Although the goodness-of-fit are equivalent for both the fits, either one supports the existence of a $p \bar{p}$ molecule-like state or bound state with greater than $7 \sigma$ significance.

\section{Summary}

Heavy flavor production are generally well described by pQCD, although the theoretical uncertainties are quite large at low $p_{T}$, especially in the case of charm production. On the other hand, quarkonium production mechanisms remain a puzzle and call for further investigations. The LHC 

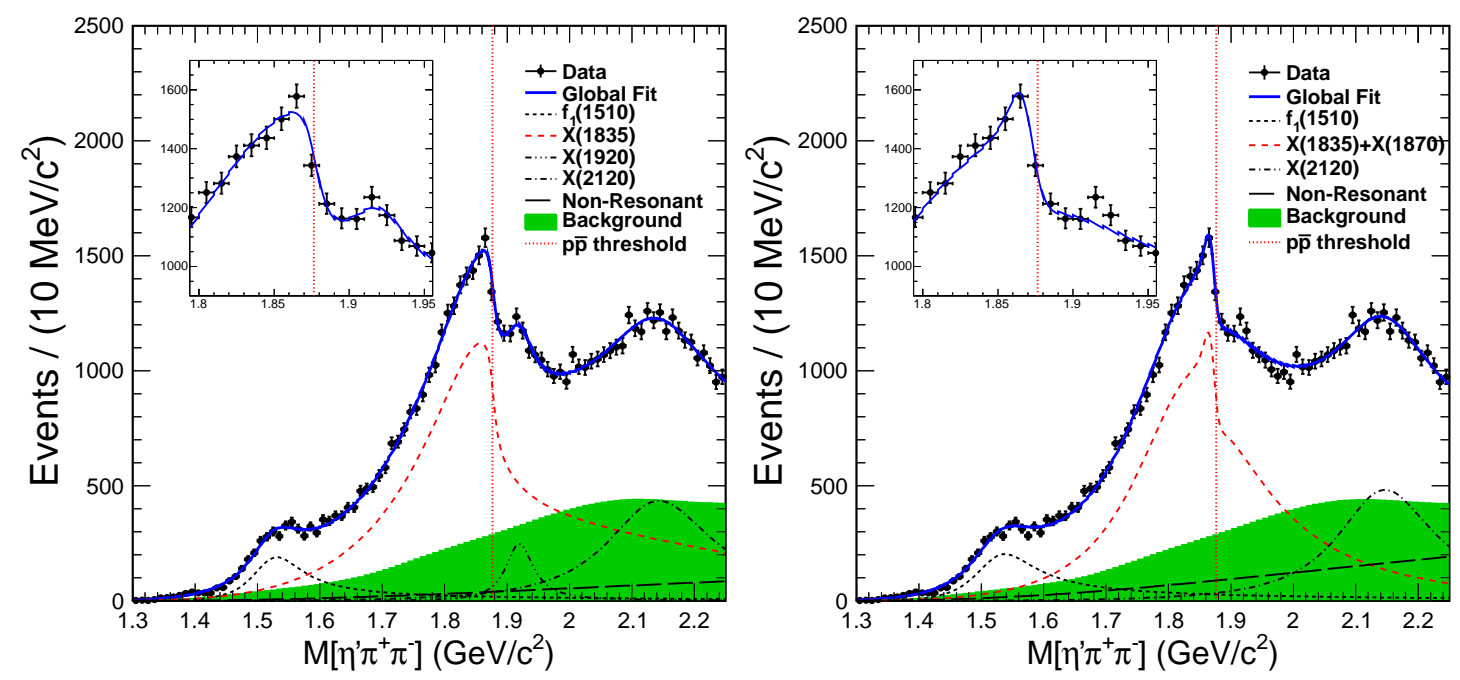

Figure 9: An anomalous line shape of the $\eta^{\prime} \pi^{+} \pi^{-}$mass spectrum near the $p \bar{p}$ mass threshold in $J / \psi \rightarrow$ $\gamma \eta^{\prime} \pi^{+} \pi^{-}$. (a) shows the fitting results with Flatté formula and (b) shows the fitting results with the coherent sum of two Breit-Wigner amplitudes

Run 2 will provide more precise measurements and strong constraints to the theoretical calculations and further understanding on the production mechanisms.

The series of discoveries of candidates of QCD exotica have expanded our knowledge of hadrons and inspired a new field of the experimental and theoretical research. No compelling theoretical picture has yet been found that provides a global description of what is seen. Further investigations with more production and decay mechanism and search for the partners with similar configuration are required to understand their nature, especially for those near thresholds. Many of the experiments producing these new results are still currently active, while a number of new experiments are on the horizon. We can expect a continuous flow of interesting experimental results and new insights into QCD in the confinement regime.

\section{References}

[1] CMS Collaboration [CMS Collaboration], CMS-PAS-BPH-15-004.

[2] R. Aaij et al. [LHCb Collaboration], JHEP 1510, 172 (2015).

[3] CMS Collaboration [CMS Collaboration], CMS-PAS-BPH-15-005.

[4] The ATLAS collaboration, ATLAS-CONF-2015-030.

[5] H. Han, Y. Q. Ma, C. Meng, H. S. Shao, Y. J. Zhang and K. T. Chao, Phys. Rev. D 94, no. 1, 014028 (2016).

[6] LHCb-paper-2016-031, in preparation

[7] M. Aaboud et al. [ATLAS Collaboration], arXiv:1610.09303 [hep-ex].

[8] R. F. Lebed, R. E. Mitchell and E. S. Swanson, arXiv:1610.04528 [hep-ph].

[9] R. Aaij et al. [LHCb Collaboration], Phys. Rev. Lett. 115 (2015) 072001. 
[10] R. Aaij et al. [LHCb Collaboration], Phys. Rev. Lett. 117, no. 8, 082003 (2016) Addendum: [Phys. Rev. Lett. 117, no. 10, 109902 (2016)].

[11] T. Aaltonen et al. [CDF Collaboration], Phys. Rev. Lett. 102, 242002 (2009.

[12] V. M. Abazov et al. [D0 Collaboration], Phys. Rev. D 89, no. 1, 012004 (2014).

[13] S. Chatrchyan et al. [CMS Collaboration], Phys. Lett. B 734, 261 (2014).

[14] R. Aaij et al. [LHCb Collaboration], Phys. Rev. D 85, 091103 (2012).

[15] J. P. Lees et al. [BaBar Collaboration], Phys. Rev. D 91, no. 1, 012003 (2015).

[16] T. Aaltonen et al. [CDF Collaboration], arXiv:1101.6058 [hep-ex].

[17] R. Aaij et al. [LHCb Collaboration], arXiv:1606.07895 [hep-ex].

[18] R. Aaij et al. [LHCb Collaboration], arXiv:1606.07898 [hep-ex].

[19] M. Ablikim et al. [BESIII Collaboration], arXiv:1611.01317 [hep-ex].

[20] [BESIII Collaboration], arXiv:1610.07044 [hep-ex].

[21] M. Ablikim et al. [BESIII Collaboration], Phys. Rev. Lett. 114, no. 9, 092003 (2015).

[22] M. Ablikim et al. [BESIII Collaboration], Phys. Rev. D 91, no. 11, 112005 (2015).

[23] M. Ablikim et al. [BESIII Collaboration], Phys. Rev. D 93, no. 1, 011102 (2016).

[24] M. Ablikim et al. [BESIII Collaboration], Phys. Rev. Lett. 110, 252001 (2013).

[25] Z. Q. Liu et al. [Belle Collaboration], Phys. Rev. Lett. 110, 252002 (2013).

[26] T. Xiao, S. Dobbs, A. Tomaradze and K. K. Seth, Phys. Lett. B 727, 366 (2013).

[27] M. Ablikim et al. [BESIII Collaboration], Phys. Rev. Lett. 111, no. 24, 242001 (2013).

[28] M. Ablikim et al. [BESIII Collaboration], Phys. Rev. Lett. 112, no. 2, 022001 (2014).

[29] M. Ablikim et al. [BESIII Collaboration], Phys. Rev. D 92, no. 9, 092006 (2015).

[30] M. Ablikim et al. [BESIII Collaboration], Phys. Rev. Lett. 112, no. 13, 132001 (2014).

[31] M. Ablikim et al. [BESIII Collaboration], Phys. Rev. Lett. 115, no. 11, 112003 (2015).

[32] M. Ablikim et al. [BESIII Collaboration], Phys. Rev. Lett. 115, no. 22, 222002 (2015).

[33] M. Ablikim et al. [BESIII Collaboration], Phys. Rev. Lett. 113, no. 21, 212002 (2014).

[34] M. Ablikim et al. [BESIII Collaboration], Phys. Rev. Lett. 115, no. 18, 182002 (2015).

[35] D. Santel et al. [Belle Collaboration], Phys. Rev. D 93, no. 1, 011101 (2016).

[36] R. Mizuk et al. [Belle Collaboration], Phys. Rev. Lett. 117, no. 14, 142001 (2016).

[37] A. Abdesselam et al., arXiv:1609.08749 [hep-ex].

[38] A. Bondar et al. [Belle Collaboration], Phys. Rev. Lett. 108, 122001 (2012)

[39] A. Garmash et al. [Belle Collaboration], Phys. Rev. D 91, no. 7, 072003 (2015)

[40] A. Garmash et al. [Belle Collaboration], Phys. Rev. Lett. 116, no. 21, 212001 (2016)

[41] V. M. Abazov et al. [D0 Collaboration], Phys. Rev. Lett. 117, no. 2, 022003 (2016)

[42] D0 Note 6488-CONF 
[43] R. Aaij et al. [LHCb Collaboration], Phys. Rev. Lett. 117, no. 15, 152003 (2016)

[44] CMS Collaboration [CMS Collaboration], CMS-PAS-BPH-16-002.

[45] M. Ablikim et al. (BES Collaboration), Phys. Rev. Lett. 95, 262001 (2005).

[46] M. Ablikim et al. (BESIII Collaboration), Phys. Rev. Lett. 106, 072002 (2011).

[47] M. Ablikim et al. (BESIII Collaboration), Phys. Rev. Lett. 115, 091803 (2015).

[48] J. Z. Bai et al. (BES Collaboration), Phys. Rev. Lett. 91, 022001 (2003).

[49] M. Ablikim et al. (BESIII Collaboration), Chin. Phys. C 34, 421 (2010).

[50] J. P. Alexander et al. (CLEO Collaboration), Phys. Rev. D 82, 092002 (2010).

[51] M. Ablikim et al. (BESIII Collaboration), Phys. Rev. Lett. 108, 112003 (2012).

[52] M. Ablikim et al. [BESIII Collaboration], Phys. Rev. Lett. 117, no. 4, 042002 (2016). 